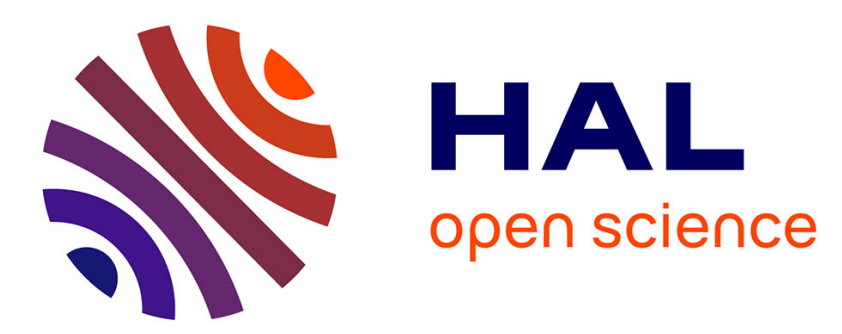

\title{
Information Topological Characterization of Periodically Correlated Processes by Dilation Operators
}

\author{
Guillaume Bouleux, Maël Dugast, Eric Marcon
}

\section{To cite this version:}

Guillaume Bouleux, Maël Dugast, Eric Marcon. Information Topological Characterization of Periodically Correlated Processes by Dilation Operators. IEEE Transactions on Information Theory, 2019, 65 (10), pp.6484-6495. 10.1109/TIT.2019.2923217 . hal-02152069

\section{HAL Id: hal-02152069 \\ https://hal.science/hal-02152069}

Submitted on 11 Jun 2019

HAL is a multi-disciplinary open access archive for the deposit and dissemination of scientific research documents, whether they are published or not. The documents may come from teaching and research institutions in France or abroad, or from public or private research centers.
L'archive ouverte pluridisciplinaire HAL, est destinée au dépôt et à la diffusion de documents scientifiques de niveau recherche, publiés ou non, émanant des établissements d'enseignement et de recherche français ou étrangers, des laboratoires publics ou privés. 


\title{
Information Topological Characterization of Periodically Correlated Processes by Dilation Operators
}

\author{
Guillaume Bouleux, Maël Dugast, and Eric Marcon
}

\begin{abstract}
Giving process information through spectral considerations has been tackled for decades. We propose in this work a new way of dealing with such an objective by giving hidden information topology of the spectral measure of non-stationary and periodically correlated processes. We used first the Kolmogorov decomposition which is a natural extension of the Naimark operator theory to obtain a sequence of rotation matrices called the dilation matrices. These matrices carry all the spectral information of the process and belong to $\mathrm{SO}(\mathrm{n})$ or $\mathrm{SU}(\mathrm{n})$ with respect to respectively the real or complex nature of the periodically correlated processes studied. In order to give a topological interpretation of the positioning of these matrices on the space of rotation matrices, we have applied a persistent homology technique and next exposed fundamental attributes. We showed that different types of periodically correlated processes are endowed with a point cloud structure that can be easily discriminated by topological and information features.
\end{abstract}

Index Terms-Dilation Theory, Non-stationary Processes, Topological Data Analysis, persistent homology, Information Entropy, Signal Analysis, Signal Classification, Betti numbers, Geometry.

\section{INTRODUCTION}

Information contained in a non-stationary stochastic process has been for example exhibited and treated by the harmonic analysis field since many decades now. Classical time-scale/time-frequency analysis [1], [2] or Fourier-like representation when the process belongs to the periodically correlated (PC) subclass [3], [4] are some well-known approaches to fulfill this purpose. They help in giving information on the underlying measure of the non-stationary process. Giving process information has also been tackled in the Information Geometry context [5], [6], [7], [8] for which the geometrical structure of the process measure is of interest and not only the geometrical structure of the process realization. Some authors have also characterized the topological structure of Information by theoretically demonstrating

Univ Lyon, INSA Lyon, DISP, EA 4570, F-69621, Villeurbanne, France \{mael.dugast,guillaume.bouleux,eric.marcon\}@insa-lyon.fr its homological property [9], [10]. In this work, we intend to give a topological characterization for nonstationary process measure.

With the results of [11] for non-stationary processes, the process' spectral measure is represented by several dilation operators. Each of them is a real or complex rotation matrix, depending on the nature of the process studied. The spectral measure of the non-stationary process is then embedded onto either the Special Orthogonal group $\mathrm{SO}(\mathrm{n})$ of real rotation matrices or the Special Unitary group $\mathrm{SU}(\mathrm{n})$ of complex rotation matrices and is represented by a sampled curve on that group. In [8], non-stationary processes are differentiated by comparing curves of dilation operators trough Square Root Velocity (SRV) functions developments [7], [12] on Lie group but nothing is mentioned on the intrinsic shape, say the topology of such curves. This is a major point we are dealing with in the present work. To our knowledge, not only there is no previous work which represents nonstationary processes and specifically $\mathrm{PC}$ processes by their dilation operators on Lie group but no previous work has brought information of the topology of the associated spectral measure.

The topological study of closed curves on a manifold $\mathcal{M}$ is usually addressed by the means of homotopy theory through the fundamental group, $\pi_{1}(\mathcal{M})$. Roughly speaking, a non-trivial fundamental group exhibits the number of times a closed curve winds. This yields to a topological invariant number called the Chern number. Problem is to catch the number of windings and even to be sure not to miss some of them. To overcome this difficulty, the cell structure of the fundamental group is exploited to give rise to homology group theory. For a concrete and a real application perspective, homology has been largely computed by means of the persistent homology. As an example, [13] shows how persistent homology can be used in linguistic, [14] reveals some topological signature of random matrices, and topological description of stochastic processes has been undertaken by [15] through dimensionality reduction techniques. In this context, recent evidence highlights the importance of 
persistent homology within the field of stochastic signal processing. This approach is adapted in consequence to the dilation matrices for giving information on their algebraic topology.

This paper begins with the section II which lays out the theoretical dimensions of the Dilation theory. This section intends to give the reader enough elements for understanding how the spectral measure of a process can be embedded in a manifold. Then, section III starts by giving an intuitive explanation for introducing the complex topic of homology. A more theoretical but not necessary part is next proposed to formally expose the homology. This section nevertheless gives the key for understanding how topological invariant shall be computed through the dilation matrices. Further, section IV introduces persistent homology and the associated persistent diagrams. Section V exposes the results obtained and proposes some interpretation and early conclusions on the topological features determined. Finally, a conclusion is drawn in section VI.

\section{EMBEDDING SPECTRAL MEASURES ON A MANIFOLD}

Even before we can talk about topological information, it is necessary to understand how the random structure which defines a stochastic process can be embedded onto a manifold. The objective of this part, therefore, is to give the reader enough elements so that he can understand this connection. The general idea is to represent the spectral measure of the process by general rotation matrices which encode the rotations necessary for the Gram-Schmidt orthogonalization of the canonical basis defined by the set $\left\{E_{i}\right\}_{i=0}^{n-1}$ of vectors with a one at the $i$-th position and zero elsewhere in the space spanned by the kernel of the random process.

\section{A. From spectral measure, shift operator to dilation operator}

We start our development by adopting the approach of [16]. In this paper, the dilation of the spectral measure is presented in the following way. Let $\left\{X_{t}\right\}_{t \in T}$ be a stationary process living on a group $T$, there is a unitary representation $U$ of $T$ on $\mathcal{H}_{X}=s p\left\{X_{t}, t \in T\right\}$, called the time-domain [17], [18], [4], [19] and such that $X_{t}=$ $U_{t} X_{e}$, where $e$ is identity element of the group $T$. In this context the operator $U$ is named the shift operator, it describes how to go from any vector $X_{t-1}$ of $\mathcal{H}_{X}$ to $X_{t}$. With now $G$ the dual of $T$, the Fourier transform of the spectral measure $F$ of $\left\{X_{t}\right\}_{t \in T}$ is given by

$$
R_{\tau}=\int_{G}[\tau, g] F(d g), \tau \in T
$$

where $[\tau, g]$ is the value of a character $g$ on $\tau$. When $T=$ $\mathbb{R}$, the characters are the functions $g: x \longmapsto e^{2 k \pi x}, k \in$ $\mathbb{R}, G$ is isomorphic to $\mathbb{R}$ and we obtain the usual Fourier transform. Finally, there exists a Hilbert space $\mathcal{H}=\mathcal{H}_{X}$ and a unitary representation $U_{g}$ such that

$$
\begin{aligned}
R_{\tau}=\left\langle X_{\tau}, X_{e}\right\rangle & =\left\langle U_{\tau} X_{e}, X_{e}\right\rangle \\
& =X_{e}^{*} U_{\tau} X_{e}=\int_{G}[\tau, g] X_{e}^{*} E(d g) X_{e}
\end{aligned}
$$

where $E$ is the spectral measure of $U_{g}$. By the unicity of the Fourier transform we have consequently

$$
F(\Delta)=X_{e}^{*} E(\Delta) X_{e} \Delta \in \Sigma
$$

with $\Sigma$ is a fixed $\sigma$-algebra of $T^{*}$. This expression finally means that the spectral measure of the process $\left\{X_{t}\right\}_{t \in T}$ in embedded in a larger spectral measure at the top left corner such that

$$
E(\Delta)=\left(\begin{array}{cc}
F(\Delta) & * \\
* & *
\end{array}\right),
$$

$E(\Delta)$ is therefore the spectral dilation of $F(\Delta)$. We recognize here the Naimark dilation, which can also be formulated through an operator viewpoint by the following fundamental theorem

Theorem 1 (Naimark Dilation). A sequence of operators $\left\{R_{n}\right\}_{n \in \mathbb{N}}$ on a Hilbert space $\mathcal{H}$ is positive definite, i.e. $\sum_{0,0}^{\infty, \infty}\left\langle R_{i-j} h_{i}, h_{j}\right\rangle \geq 0$ for all sequences $\left\{h_{i}\right\}_{0}^{\infty}$ if and only if there exits an isometry $U$ on $\mathcal{K} \subset \mathcal{H}$ such that

$$
R_{i j}=P_{\mathcal{H}} U_{\mid \mathcal{H}}^{j-i}
$$

where $P_{\mathcal{H}}$ stands for the orthogonal projection on $\mathcal{H}$ and $\mid \mathcal{H}$ indicates a dimension reduction to $\mathcal{H}$. In addition, if

$$
\mathcal{K}_{R}=\bigvee_{n \geq 0} U^{n} \mathcal{H}_{n}
$$

then $U$ is unique up to an isomorphism.

Basically (5) implies that the whole correlation sequence is obtained by successive power of the unitary dilation operator $U$. Actually, by bringing (2) and (5), we see that the unitary dilation operator $U$ is nothing but the shift operator. When digging in the literature, we found many references dealing with the use of the Naimark theorem for stochastic processes characterization. For example, [20] or [21] tackle the dilation theory by spectral measure considerations whereas [22], [23], [24], [25] uses the positive definite sequences as a starting point for developing the Naimark dilation. Finally, the readers will find explicit proofs for the correspondance between the shift operator and the Naimark dilation in [26] or [27]. 
We finish this part by giving the structure of the unitary dilation when a process is nonstationary. In this scenario, the sequence of operators of Theorem 1 is no more explained by only one unique unitary operator $U$. Actually, it is enough to think that for each time of origin there exists a specific unitary dilation. This is the concept of nonstationarity. The statistics of the process do not depend on just of the lag but also of the time they are computed. At each time, there is a different spectral component, which yields to different unitary dilations. By keeping the same notation adopted for the time domain definition of the shift operator, at each time there is a new shift operator. Then, $X_{t}=U_{t} X_{t-1}=\prod_{i=0}^{t} U_{i} X_{e}$. The formal generalization of the Naimark unitary dilation is thus given by the Kolmogorov decomposition which writes

Theorem 2 (Kolmogorov Decoposition). Let $R$ a positive definite kernel. Then, there is a Hilbert space $\mathcal{H}$ and applications $U_{i}, i \in \mathbb{Z}$ such that

$$
\begin{aligned}
R_{i, j} & =\left.P_{\mathcal{H}} U_{i} U_{i-1} \cdots U_{j-1}\right|_{\mathcal{H}} \forall i, j \in \mathbb{N} \\
\mathcal{K}_{R} & =\bigvee_{n \in \mathbb{Z}} V^{n} \mathcal{H}_{n}
\end{aligned}
$$

with $V^{i}=\prod_{i=0}^{i-1} U_{i}$. A sequence of operator is then obtained by the product of unitary dilations $U_{i}, i \in \mathbb{Z}$.

\section{B. Computation of the dilation matrix}

So far, the appearance of the Naimark dilation operator seemed rather theoretical, but the works of [11], [28], [29], [24] and latter [30], [31] have shown how to obtain a computational expression of the unitary dilation $U$ associated with the process $\left\{X_{t}\right\}_{t \in T}$. Two major points are to be recalled for the obtention of $U$. The first one is to remind the one-to-one correspondence between the correlation (assumed normalized) matrix $R$ of $\left\{X_{t}\right\}_{t \in T}$ and a sequence of partial correlation coefficients, the parcors. This one-to-one correspondence has been largely tackled in the literature by many different faces; some have an interest in the polynomial decomposition of random measure [32], other on geometrical characteristics of the spectral measure [33], [5], [7]. Among all the approaches bring out by this vaste literature, we found that the definite positive property of the correlation matrix $R$ gave somewhat the most easy to interpret oneto-one correspondence, this is deeply explained in [11]. In this reference, is associated to the correlation matrix $R$ another matrix, let's say $\Gamma$ which has the definition

$$
\Gamma=\left(\begin{array}{ccccccc}
0 & \Gamma_{1,2} & \Gamma_{1,3} & \ldots & \ldots & \Gamma_{1, n} & \ldots \\
& 0 & \Gamma_{2,3} & \Gamma_{2,4} & \ldots & \Gamma_{2, n} & \ldots \\
& & \ddots & & \ddots & & \ldots \\
& & & & & \Gamma_{n-1, n} & \ldots
\end{array}\right) .
$$

We arrive now at the second major points. Since by definition $\left|\Gamma_{i, j}\right| \leq 1, \forall j>i, i=1, \ldots, n$, all the parcors are also considered as contractions. This is a fundamental point which allows to use the following theorem given in [16]

Theorem 3. [Dilation of a Contraction] For every contraction $T$ on the Hilbert space $\mathcal{H}$ there exists a unitary dilation $U$ on a space $\mathcal{K}$ containing $\mathcal{H}$ as a subspace, which is minimal, that is, such that

$$
\mathcal{K}=\bigvee_{-\infty}^{\infty} U^{n} \mathcal{H}
$$

This minimal unitary dilation is determined up to isomorphism, and thus can be called the minimal unitary dilation of $T$.

Owing to Theorem 3, this yields to explicitly express the dilation of the contraction $T=\Gamma_{i, j}, j>i, i=$ $1, \ldots, n \in \mathcal{H}$ as

$$
U=\left(\begin{array}{cc}
T & D_{T^{*}} \\
D_{T} & -T^{*}
\end{array}\right) \in \mathcal{K}=\mathcal{H} \oplus \mathcal{H}
$$

where $D_{T}=\left(I-T^{*} T\right)^{1 / 2}$ is called the defect operator. The basic structure of (9) stands for the Julia operator which becomes when $\mathcal{H}=\mathbb{R}$, the so-called Givens rotation. To finally obtain the unitary dilation of the spectral measure $F$ of (4) it suffices now to compose with (9) and (7). Due to the non stationarity of $\left\{X_{t}\right\}_{t \in T}$, and as defined by the Kolmogorov decomposition introduced in (2), each sub-unitary dilation $U_{i}$ are obtained by applying successive Givens rotations on the rows of (7) (please refer to [11] for a complete proof), such that

$$
U_{i}=G\left(\Gamma_{i, i+1}\right) G\left(\Gamma_{i, i+2}\right) \cdots G\left(\Gamma_{i, n}\right)
$$

where $G\left(\Gamma_{i, i+k}\right)$ is the Givens rotation of $\Gamma_{i, i+k}$ defined as

$$
G\left(\Gamma_{i, i+k}\right)=I \oplus\left(\begin{array}{cc}
\Gamma_{i, i+k} & D_{\Gamma_{i, i+k}^{*}} \\
D_{\Gamma_{i, i+k}} & -\Gamma_{i, i+k}^{*}
\end{array}\right) \oplus I
$$

which explicitly writes in matrix form as

$$
\left(\begin{array}{ccccccc}
\ddots & & & & & & \\
& I & & & & & \\
& & I & & & & \\
& & & \Gamma_{i, i+k} & D_{\Gamma_{i, i+k}^{*}} & & \\
& & & D_{\Gamma_{i, i+k}} & -\Gamma_{i, i+k}^{*} & & \\
& & & I & & \\
& & & & I & \\
& & & & & \ddots
\end{array}\right) .
$$

We draw the attention of the reader to the necessity to have the same number of parcors to build each Givens 


$$
U_{i}=\left(\begin{array}{ccccc}
\Gamma_{i, i+1} & D_{\Gamma_{i}^{*}} \Gamma_{i+1} & D_{\Gamma_{i, i+1}^{*}} D_{\Gamma_{i, i+2}^{*}} \Gamma_{i, i+3} & D_{\Gamma_{i, i+1}^{*}} D_{\Gamma_{i, i+2}^{*}} D_{\Gamma_{i, i+3}^{*}} \Gamma_{i, i+4} & \cdots \\
D_{\Gamma_{i, i+1}} & -\Gamma_{i, i+1}^{*} \Gamma_{i, i+2} & -\Gamma_{i, i+1}^{*} D_{\Gamma_{i, i+2}^{*}} \Gamma_{i, i+3} & -\Gamma_{i, i+1}^{*} D_{\Gamma_{i, i+2}^{*}} D_{\Gamma_{i, i+3}^{*}} \Gamma_{i, i+3} & \ldots \\
0 & D_{\Gamma_{i, i+2}} & -\Gamma_{i, i+2}^{*} \Gamma_{i, i+3} & -\Gamma_{i, i+2}^{*} D_{\Gamma_{i, i+3}^{*}} \Gamma_{i, i+4} & \ldots \\
0 & 0 & D_{\Gamma_{i, i+3}} & -\Gamma_{i, i+3}^{*} \Gamma_{i, i+4} & \ldots \\
0 & 0 & 0 & D_{\Gamma_{i, i+4}} & \ldots \\
\cdot & \cdot & . & . & \ldots \\
\cdot & . & . & . & \ldots
\end{array}\right)
$$

rotation. Finally, a basic form for the sub-unitary dilation matrix $U_{i}$ is therefore (13) which is often called an upper Hessenberg matrix in the literature [34]. This is a rotation matrix which belongs to $\mathrm{SO}(\mathrm{n})$ or $\mathrm{SU}(\mathrm{n})$, the special orthogonal of special unitary group respectively.

\section{A step by step implementation example}

To illustrate these theoretical results, we have generated five periodically correlated processes and displayed their $\mathrm{SO}(3)$ representation, thus two parcors were used for each of the sub-unitary dilation matrix $U_{i}$. Given the examples of Fig.1, for which the periodicity of each signal is equal to $T=20$ samples, we have used the perARMA $\mathrm{R}$ package and the included function perpacf to compute the 20 sequences of (arbitrary) 3 parcors. Indeed, we have basically $X_{t+T}=U_{t} X_{e}$ for a periodically correlated process with period $T$; only $T$ sequences bring the spectral information. Estimating 3 parcors yield to a representation of the process on $\mathrm{SO}(4)$, for the representation of Fig. 1 we kept only 2 parcors so. We use next the set $\left\{\Gamma_{i, j}\right\}_{i=1, j=1}^{i=20, j=3}$ to obtain each subunitary dilation matrix $U_{i}$ with respect to (14).

The unitary dilation $U_{i}$ of $\left\{X_{t}\right\}_{t \in T}$ (for the general nonstationary case) are then embedded in the Lie group of real or complex rotations. As, in addition we assume that $\left\{X_{t}\right\}_{t \in T}$ is periodically correlated, the set of subunitary dilation $\left\{U_{i}\right\}_{i=1, \ldots, n}$ is periodic as well and the spectral measure $F$ draws a closed path on the Lie group. Since now the process $\left\{X_{t}\right\}_{t \in T}$ has a representation on a differential manifold, we are able to give its topological intrinsic characteristics.

\section{HOMOLOGY}

The fundamental previous section has defined a way to embed a nonstationary stochastic process on a Lie group. The process is represented by points in this manifold, and the set of points draw a closed path. We aim now at extracting topological invariant from this shape.

This section is devoted then to the presentation of topology and algebraic topology tools that will be used to extract intrinsic topological information from the periodically correlated processes studied. We will first introduce the basic concepts of homology and a theoretical part will follow for people who would be interested in a more mathematical justification. With the basis of homology in mind, the second part can be omitted without compromising the understanding of the results.

\section{A. The short basis of homology}

The very basis of homology is to characterize ndimensional shapes (let's say discrete shapes) by their k-dimensional holes, $k<n$. Unlike homotopy, which uses the notion of loops, i.e., a closed path that starts and ends at the same point, homology uses the notion of cycles, i.e. a closed path without the consideration of its origin. To determine the number of dimensional holes of a shape, the points are joint together to form what is called a cell in the algebraic topology context. When the cells are glued together, they form a complex, a sort of skeleton of the shape. There are several possibilities for defining a cell, and thus a complex. In order to meet our needs, we chose the simplicial complex that meets the simple interpretation and calculation objectives. The skeleton of the form is then described by dimensional simplices, whose forms are illustrated by the Fig. 3. The idea behind homology is to then look for the closed path created by the composition of dimensional simplices. To put words on this, a 1-dimensional hole, which we will associate with a circle, is characterized by the sum of the segments (1-simplicies) of the skeleton to form a closed trajectory (a cycle). If now this closed trajectory is not a part (a boundary) of another form constituted by the composition of triangles (2-simplicies), then it is asserted that the closed trajectory represents a 1dimensional hole for the shape. In that case, it belongs to the first homology group, denoted $H_{1}$ and the topological invariant associated with the number of independent 1dimensional hole for the shape is defined as the Betti number, $\beta_{1}=\operatorname{rank}\left(H_{1}\right)$. The shape is finally described by its Betti numbers $\left(\beta_{0}, \beta_{1}, \ldots, \beta_{k}\right) \forall k<n$. 


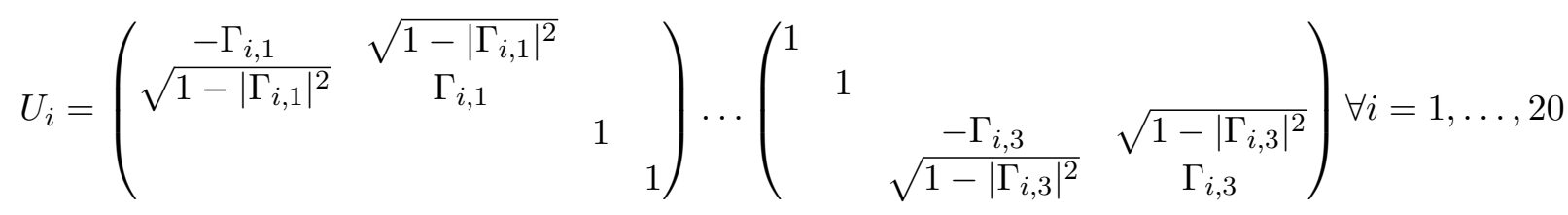

\section{B. Illustration by the example}

Let us give a simple example to illustrate these concepts. In the Fig. 3 the triangle $v=(a, b, c)$ has faces $(a b),(b c)$, and $(c a)$. The boundary of the triangle is then $(a b)+(b c)+(c a)$ which defines a closed path. Therefore, $(a b)+(b c)+(c a)$ is a cycle, but it is also a boundary. We conclude that $(a b)+(b c)+(c a)$ do not represent a hole.

To end this illustration part, we propose to the reader to observe the Fig. 4. This plot displays three discrete shapes materialized by the distribution of the points along a circle, Fig.4-(b) or a torus, Fig. 4-(c). In this example, Fig. 4-(a) is a point. It is a connected component and thus has a Betti number $\beta_{0}=1$. The Betti numbers associated with Fig. 4-(b) are $\left(\beta_{0}=1, \beta_{1}=1\right)$, there is consequently one connected component and 1dimensional hole. Finally, the Betti numbers obtained for points distributed along a torus are $\left(\beta_{0}=1, \beta_{1}=\right.$ $\left.2, \beta_{2}=1\right)$, i.e. one connected component, any closed path drawn on the surface can be reduced to a point, two circles, the intrinsic generators, and one cavity.

\section{The mathematical justification}

Consider $S$ as a set of points of $\mathbb{R}^{n}$ and fix $k \leq n$. Let for the example $\sigma=\left(v_{0}, v_{1}, \cdots, v_{k}\right)$ be the $k$ simplex made by the convex hull of its $(k+1)$ vertices. Then, a 0 -simplex is a point of $S$, a 1 -simplex is a segment joining two points of $S$, a 2 -simplex is a filled triangle, a 3-simplex is a filled tetrahedron and so on. The faces of a $k$-simplex is the sub- $k$-simplex given by $\left(v_{1}, \cdots, v_{i-1}, v_{i+1}, \cdots, v_{k}\right)$. The Fig. 3 gives visual representation for these concepts.

Definition 1 (simplicial complex). The set of $k$ simplices which are either disjoint or intersect in a common face is called a simplicial complex and is denoted $\mathcal{C}_{k}$. It is often seen as the skeletal representations (mesh type representations) of data point clouds.

We recall here that the fundamental question raised by the homology theory is to determine intrinsic dimensional-hole features of the underlying point clouds. Is to be determined how these dimensional-holes are to be found when the simplicial complex is obtained. We have to first define how $k$-simplices compose themselves.
Definition 2 ( $k$-chain). The formal sums of $k$-simplices in $\mathcal{C}_{k}$, called $k$-chain, is endowed with a group structure when we define the inner commutative addition $i+j=$ $(i \cup j)-(i \cap j)$ for all $i, j \in C_{k}$. This amount to an addition over the field $\mathbb{Z}_{2}$.

To illustrate, if two 0 -simplices $(a)$, and $(b)$ are disjoint, then $(a)+(b)=(b)+(a)$ whereas $(a)+(a)=$ $(b)+(b)=0$. Finally, it remains to know when a $k$-chain participates or not to the definition of a dimensionalhole. To proceed, we need the definition of what is called boundary and cycle.

Definition 3 (Boundaries and cycles). The boundary of a simplex $\sigma \in \mathcal{C}_{k}$ is the formal sum of the faces (of dimensions $k-1$ ) of $\sigma$. It defines a group homomorphism $\partial_{k}: C_{k} \rightarrow C_{k-1}$. An $k$-chain $u$ is then a $k$-boundary if there exists $(k+1)$-chain $v$ such that $\partial_{k+1}(v)=u$, meaning that $v \in I m \partial_{k+1}$. Now a $k$-chain $u$ is a cycle if $\partial_{k}(u)=\emptyset$, meaning that $u \in \operatorname{Ker}\left(\partial_{k}\right)$.

We can now define the $k$-th homology group, as

$$
H_{k}=\operatorname{Ker}\left(\partial_{k}\right) / \operatorname{Im}\left(\partial_{k+1}\right) .
$$

It consists of all elements that are cycles (dimensional holes) but not boundaries. By using again the example of section III-B but in a more formal way; we obtain that the boundary map $\partial_{2}$ of the triangle $v=(a, b, c)$ is $\partial_{2}(v)=(a b)+(b c)+(c a)$ and the boundary map $\partial_{1}$ is $\partial_{1}((a b)+(b c)+(c a))=(a)+(b)+(b)+(c)+(c)+(a)=$ $\emptyset$. The k-chain $u=(a b)+(b c)+(c a)$ is consequently a cycle since $\partial_{1}(u)=\emptyset$ and is a boundary as well, indeed $\partial_{2}(v)=u$. Then $\partial_{2} \circ \partial_{1}=\emptyset$ and $u$ is not an element of first homology group $H_{1}$.

\section{PERSISTENT HOMOLOGY}

Having now a better idea on the mathematical concepts of homology, we turn the discussion on the persistent homology. persistent homology has been applied for time series classification, [35], image pattern recognition [36], biology [37], phylogenetic [38], science of language [39] and various other fields like [40] or for brain monitoring [46], [47]. As seen in the previous sections, the group homology is obtained thanks to the comparison of simplices at different dimensions. The invariants, the Betti numbers for example, directly depend on the 

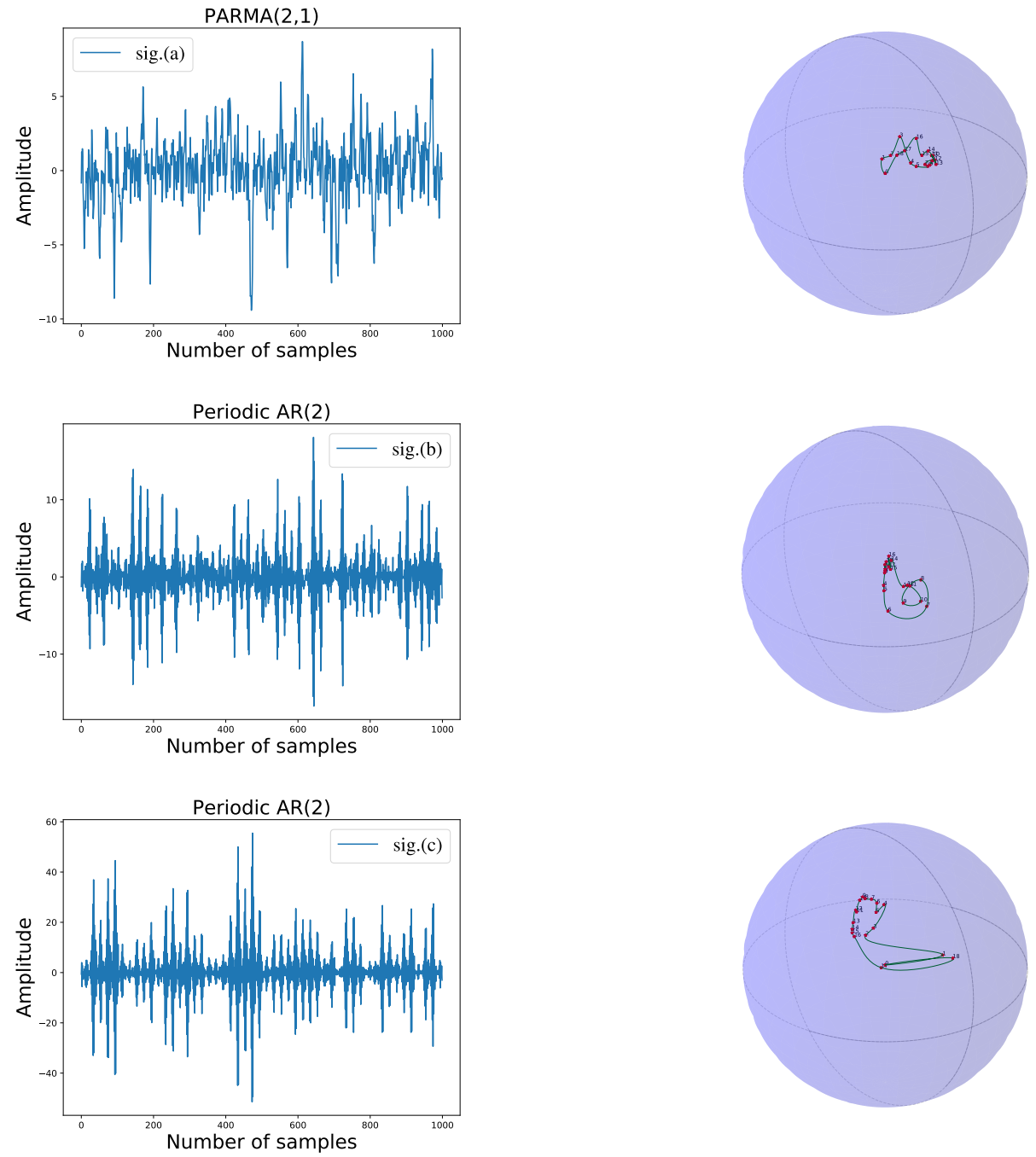

Fig. 1. (Left column), one realization of each of the three signals from the 2 classes PARMA $(2,1)$ and PAR(2). Each of these signals is periodically correlated with a period $T=20$ samples among the 1000 samples available. The sig. $(b)$ and sig.(c) have different generating coefficients. (Right column), an example of a trajectory formed by the dilation matrices in $\mathrm{SO}(3)$ for the associated signals. The trajectory is represented inside the ball of radius $\pi$. To plot the curve, we have first interpolated with splines into the Lie algebra and used next the exponential map to return on $\mathrm{SO}(3)$.

dimension of the simplicial complex so. If we dispose of $\mathrm{n}$ points and chose to build an n-dimensional complex, some hidden invariants may not be observed as cycles will necessarily be boundaries as well. A contrario, if we chose to determine the invariants with a number $k<n$ of points, high dimensional invariants could be missed. This precisely why persistent homology is used for, it computes homology at different scales, that is with varying simplicial complex dimensions, and keep track of the evolution of the homology groups. This corresponds to build a filtered complex with the sequence $\mathcal{C}_{1} \subset \mathcal{C}_{2} \cdots \subset \mathcal{C}_{n}$, with $\mathcal{C}_{n}$ the maximum simplicial complex. Once again, the literature is extremely vast about the choice to make for the filtration process. This is largely out of the scope of this paper and we let the readers interested explore more the literature for comparisons and properties of each of the filtration. For our concern, we have chosen the Vietoris-Rips filtration for it simple implementation and robustness. This method consists in fixing the dimension of the simplicial complex by the number $\rho$. The simplices $\left(v_{1}, \cdots, v_{k}\right)$ are next formed as soon as $\left\|v_{i}-v_{j}\right\|<\rho, \forall i, j$. This operation is reproduced with an increasing $\rho$ until the simplicial maximum complex containing all the data is reached. This method is briefly illustrated in Fig. 5. More details on the computation can be found in [41], [42] and [43].

During the construction of the Vietoris-Rips filtration, holes will appear and then later disappear due to the increasing dimension of the complex. This phenomenon 

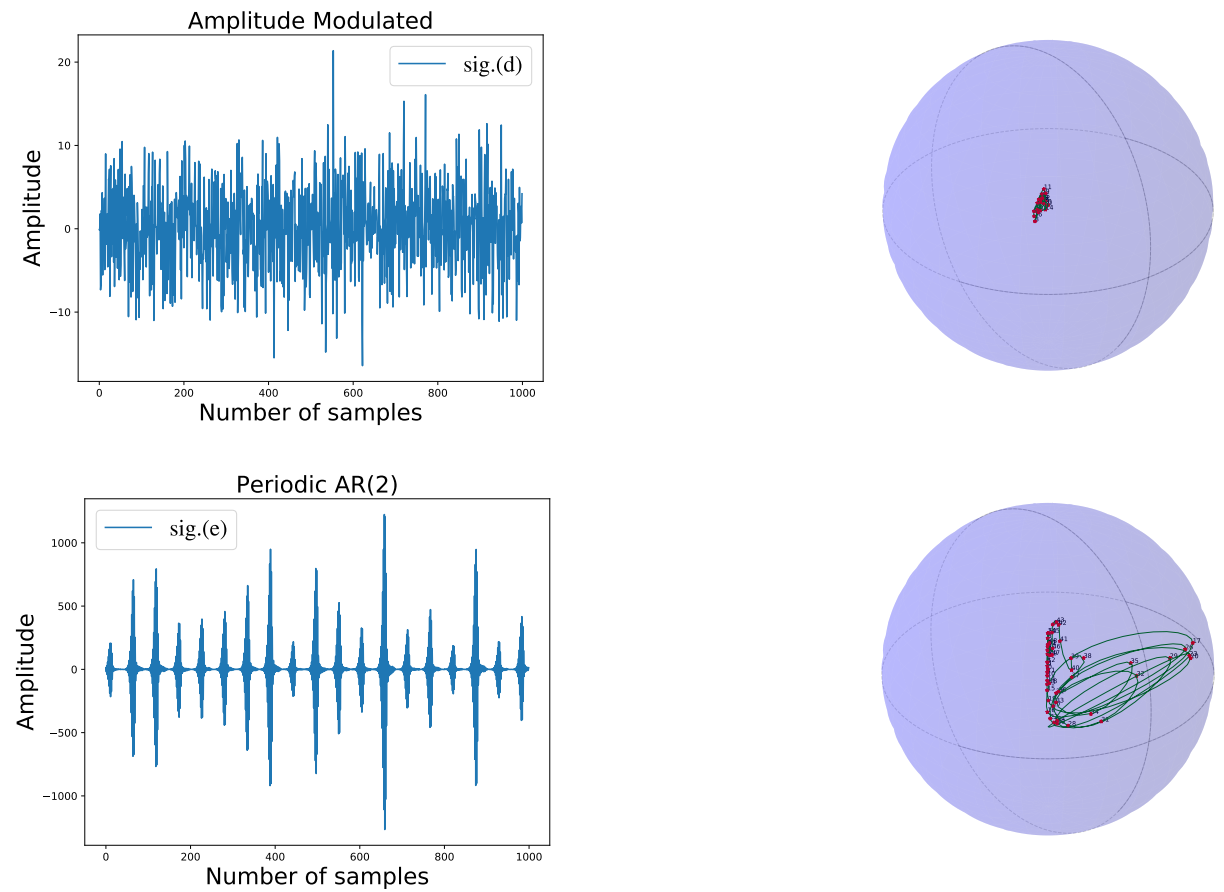

Fig. 2. (Left column), one realization of each of the 2 signals from the 2 classes PAR(2) and Amplitude Modulated. The sig.(d) is periodically correlated with a period $T=20$ samples among the 1000 samples available, and sig. $(e)$ has the same generating coefficients than that of sig.(c) but with a period of $T=54$. (Right column), an example of a trajectory formed by the dilation matrices in $\mathrm{SO}(3)$ for the associated signals. The trajectory is represented inside the ball of radius $\pi$. To plot the curve, we have first interpolated with splines into the Lie algebra and used next the exponential map to return on $\mathrm{SO}(3)$.

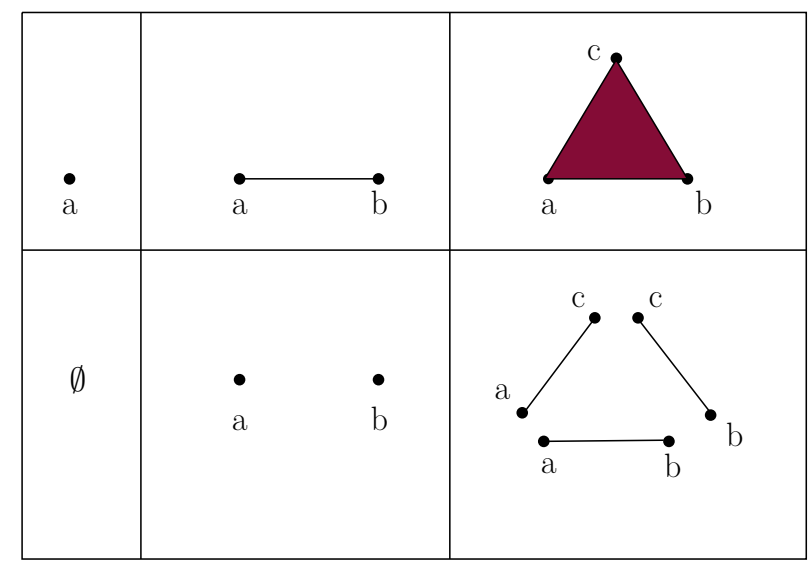

Fig. 3. A n-simplex is the convex hull of $(n+1)$ points in an $n$ dimensional space. It is the generalization of a triangle. Top (from left to right): A 0 -simplex is a point, this is (a) into the plot. A 1simplex is a segment, this is $(a, b)$ (where $a$ and $b$ are the vertices that are at the extremity of the segment), a 2-simplex is a triangle $(a, b, c)$ in the plane. Bottom (from left to right): the corresponding faces.

is reported in a diagram called a persistent diagram. It encodes the birth time and the death time of the dimensional hole. To fix ideas, we invite the reader to examine Fig. 6. This plot allows to see for three different $\rho$ the evolution of the simplicial complex and we hope illustrates why some holes appear at a certain dimension and disappear next. The corresponding persistent diagram is also provided in this plot. The toy example being one dimensional, only 0 -simplices and 1-simplicies are computed. This gives the 0 -order holes and 1 -order holes displayed by Fig. 6 . The most important result given by this figure is the lonely point at coordinates $(0.45,1.2)$. It materializes for $\rho=0.45$ the birth of a 1-dimensional hole, and the death of this hole for $\rho=1.2$, these facts are explicitly shown by Fig. 6 .

To our purpose, the shapes we would like to topologically characterize are the shapes obtained by the dilation matrices on $\mathrm{SO}(\mathrm{n})$. In order to compute the persistent homology on this Lie group with the Vietoris-Rips filtration, we have used the metric $d(A, B)=\left\|\log \left(A^{T} B\right)\right\|$, where $\|$.$\| denotes the classical Frobenius norm [44].$

These theoretical explanations done, we now propose to use the persistent homology and the underlying persistent diagram. We aim consequently at showing that according to their non-stationarity, PC processes have intrinsic topological features.

\section{THE TOPOLOGICAL INFORMATION}

This section is devoted to showing, by the analysis of the persistent diagrams, that the different periodically 

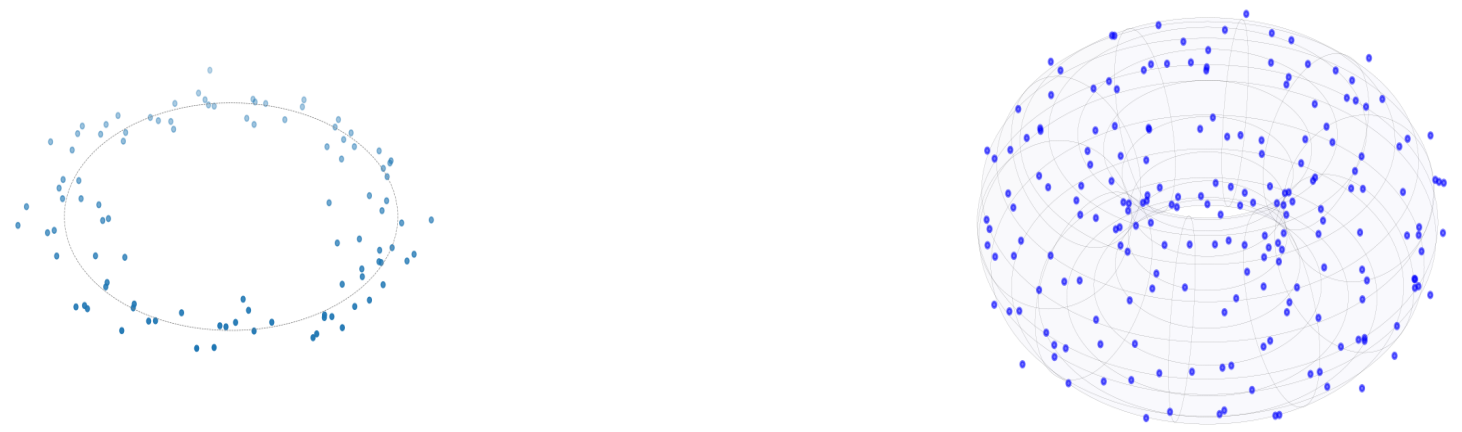

(a)

(b)

Fig. 4. (a) points distributed along with a circle, the Betti numbers are $\left(\beta_{0}=1, \beta_{1}=1\right)$, (b) points distributed along with a torus, the Betti numbers are $\left(\beta_{0}=1, \beta_{1}=2, \beta_{2}=1\right)$.

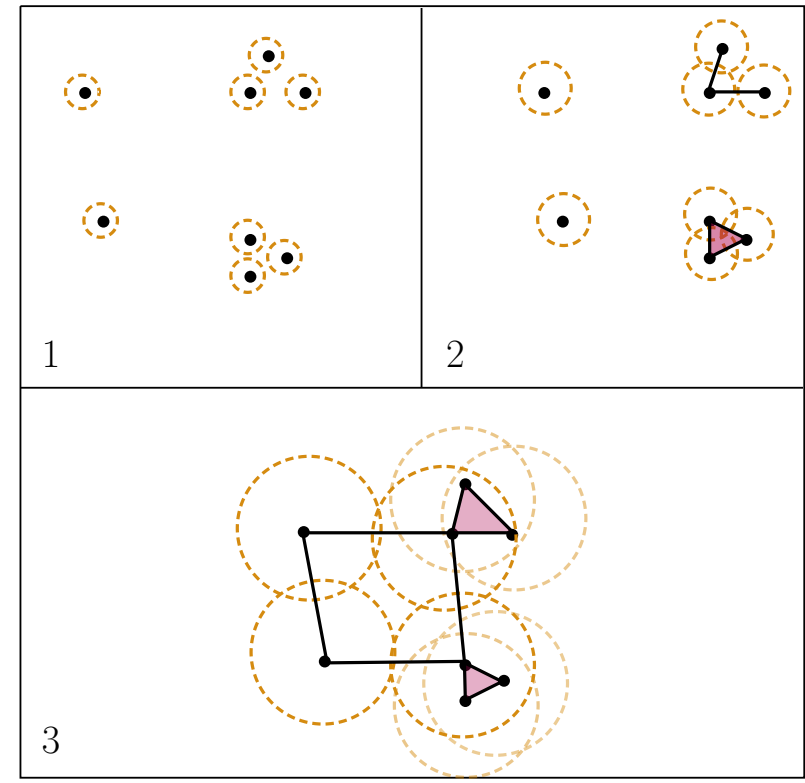

Fig. 5. A nested sequence of simplicial complexes is created by increasing the radius of balls represented in orange. As soon as two balls intersect, the points in their center are connected. When three balls intersect, we form the triangle, and so on. At stage 3 a hole appears. It is not yet the boundary of a simplex. This hole will disappear when its four vertices will be two by two connected.

correlated processes classes illustrated in Fig. 1 and Fig. 2, do have intrinsic topological features. For the experience, we have computed 500 trials of the three models proposed and computed the persistent homology on the interpolated curves. The computation of the parcors was carried out using the R package perARMA, and the python package dionysus [40] was used for the persistent diagrams computations.

\section{A. Information given by the persistent diagrams}

The Fig. 8 displays the persistent diagrams of 4 randomly selected realizations for each of the processes. The first point to raise is the no second order diagram, whatever be the class of the process. This point will be a bit more explored in the next section, but we can already say that the representation of a process on $\mathrm{SO}(3)$ yields to a curve which belongs to a preferred plane. In the context of rotation matrices, it suggests that the spectral measure of a process is oriented. The next point to raise is the relative stability of these diagrams. Often, the points are located in a closed neighborhood, it suggests that the closed path made of the dilation matrices is quite stable as well. To one trial to the other, the topological characteristics are the same. Finally, as one could have expected via the analysis of the sig. $(d)$ curve of Fig. 2, this process tends to be a stationary one. This is confirmed by Fig 8-(d) which reveals no particular hole. A contrario, the analysis of the sig.(e) of Fig. 2 suggested that there existed several loops, this is once again confirmed by the persistent diagram of Fig. 8-(e). The synthesis of these results brought out by the persistent diagrams of Fig. 8, yield us to prematurely conclude that which needs to be consolidated by other analysis. Since only 4 random trials have been showed, we propose now to analyze the average Betti number sequence of all the trials. 

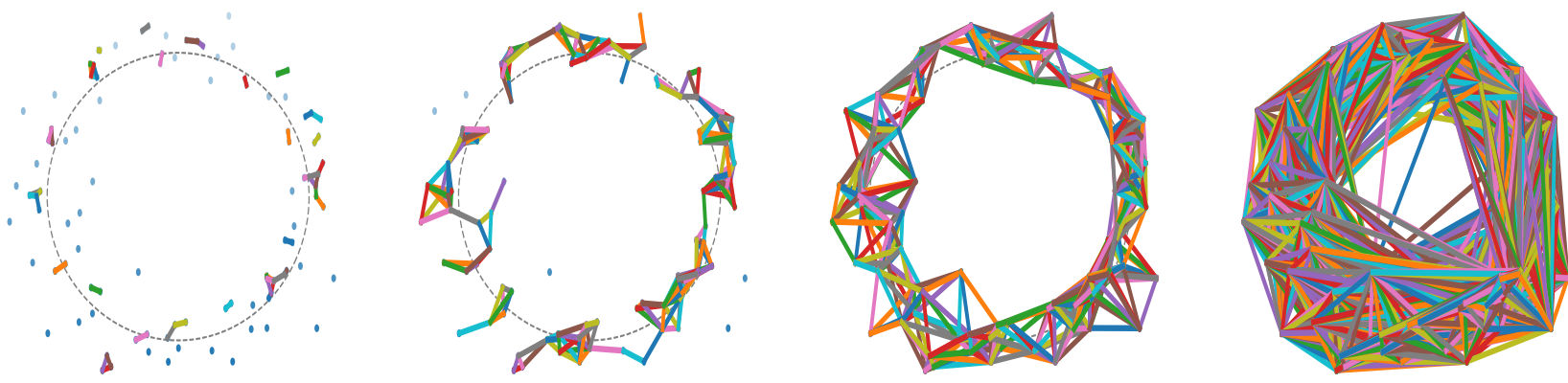

Fig. 6. From left to right, an increasing sequence of simplicial complexes for points distributed along with a circle. The number $\rho$ is the distance for the Vietoris-Rips filtration.

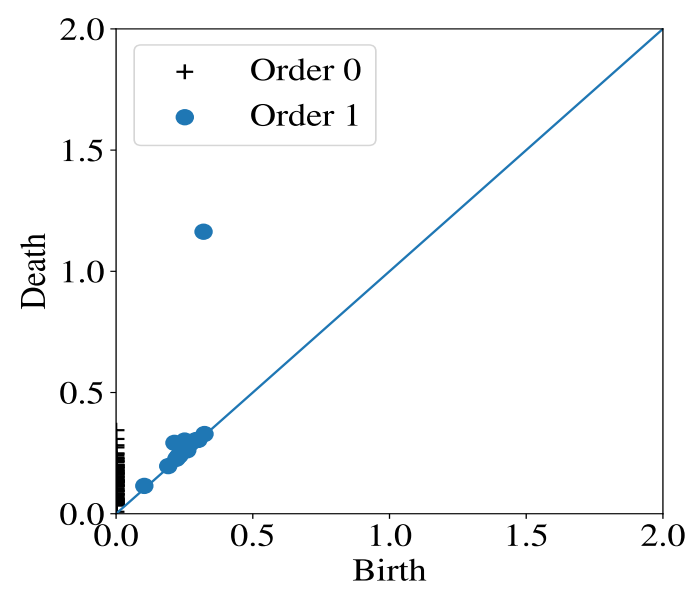

Fig. 7. Persistent diagram of the noisy circle of Fig. 6, the axes correspond to the steps $\rho$ of the filtration.

\begin{tabular}{|l||ccc|}
\hline Model & $\beta_{0}$ & $\beta_{1}$ & $\beta_{2}$ \\
\hline PARMA(2,1) & 1 & 1 & 0 \\
PAR(2), $T=20$ & 1 & 1 & 0 \\
PAR(2), $T=54$ & 4 & 2 & 0 \\
Amplitude Modulated & 0 & 0 & 0 \\
\hline \multicolumn{1}{|c|}{ TABLE } & &
\end{tabular}

BETTI NUMBERS ACCORDING TO THE CLASS OF THE PROCESSES.

\section{B. Information given by the Betti numbers}

Similarly to the approach of [14], we have analyzed the sequence of Betti numbers. We present the results in Fig. 9, for the 0-order diagram Fig. 9-(a), the 1order diagram Fig. 9-(b) and the 2-order diagram Fig. 9- (c). The different curves proposed are the averaged curves over the 500 realizations of the Betti sequences. Let us start by the end and the plot which reinforces the conclusion previously mentioned. This is, as seen previously, the Betti sequences associated with the 2order diagram. The plot of Fig. 9-(c) clearly shows that almost all the processes do not have a second order hole, so no cavity. Even if the sig.(e) admits Betti numbers variations along with the filtration, the amplitude of these variations is so weak that we can not consider the sig. (e) to have a curve which spreads off over one more dimension. More appealing is the Fig. 9-(b). This exceptional figure shows all we need to conclude on the topological features. We have an almost stationary process, this is $\operatorname{sig.}(d)$ which has consequently no loop in its representation on $\mathrm{SO}(3)$. We have two $\operatorname{PAR}(2)$ models with different generating coefficients, they are the sig. $(b)$ and sig.(c) which admits the same averaged Betti numbers sequence. We have a PAR(2) model with the same generating coefficients than that of the sig.(b) but with a period $T=54$ instead of $T=20$, this is the sig.(e). This process shows the highest number of loops in its representation and finally, we have the sig.(a) which is a PARMA $(2,1)$ model. Its averaged Betti numbers sequence is without any doubt different from the other sequences. So, there is no ambiguity in discerning the process classes, even more, if the generating coefficients are different, the averaged Betti numbers sequence says this is the same class. At least, and to 


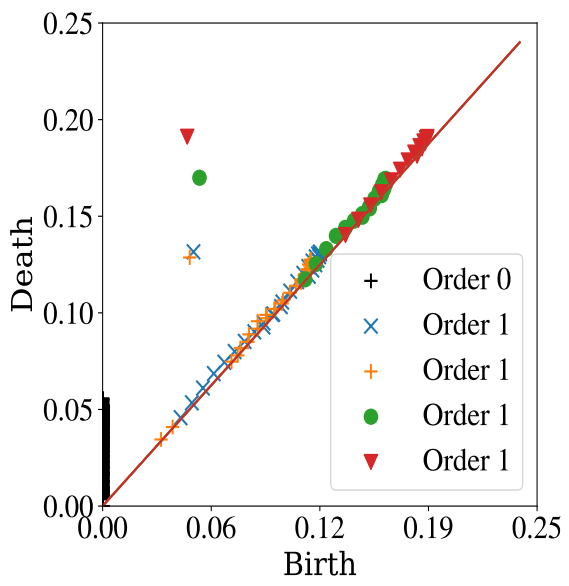

(a)

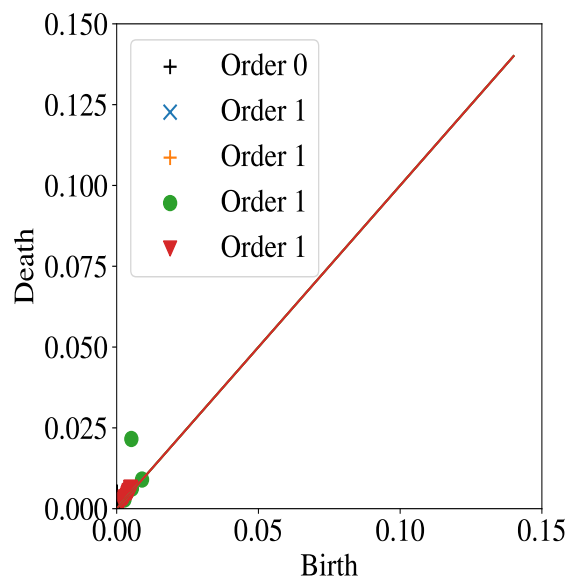

(d)

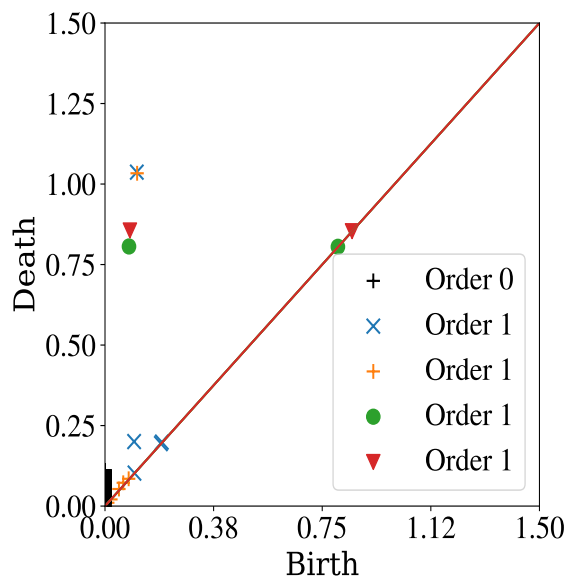

(b)

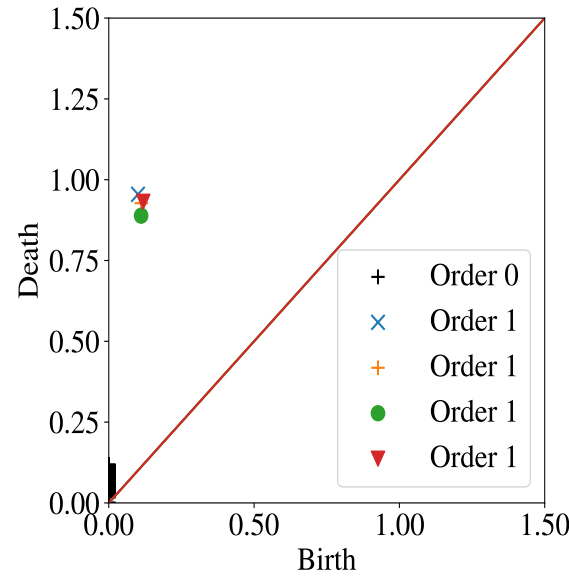

(c)

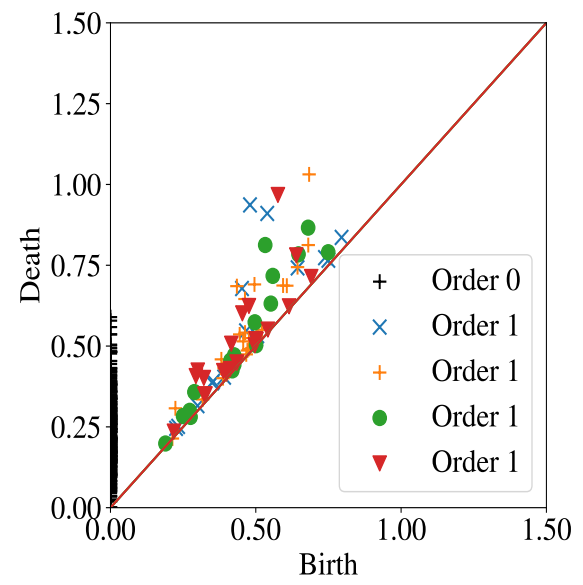

(e)

Fig. 8. Four selected at random persistent diagrams associated with (a) the sig.(a), (b) the sig.(b), (c) the sig.(c), (d) the sig.(d), and (e) the $\operatorname{sig} .(e)$.

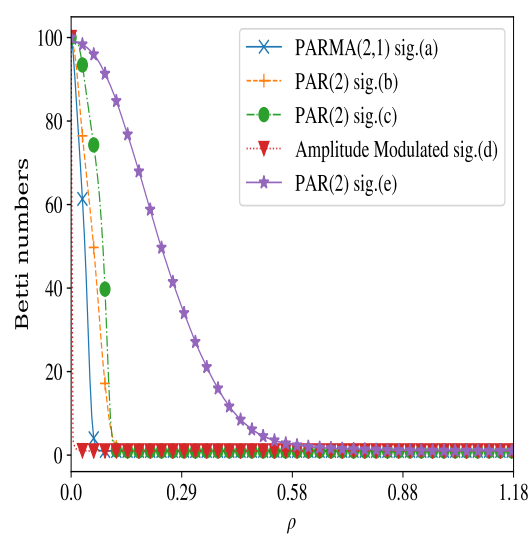

(a)

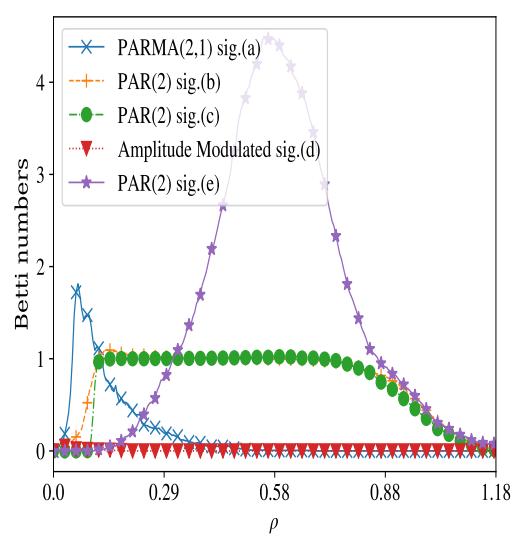

(b)

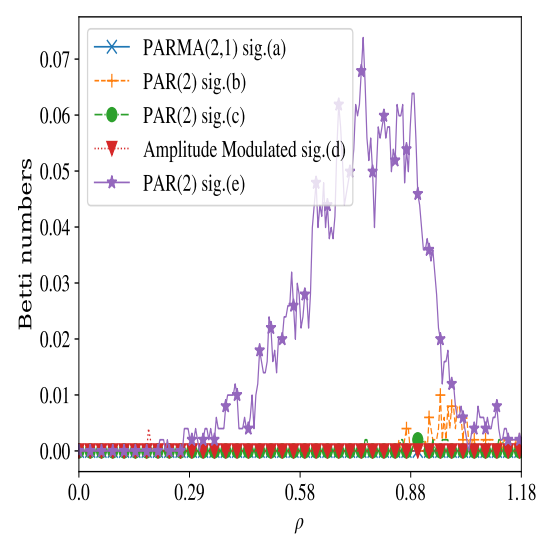

(c)

Fig. 9. Averaged Betti numbers sequences associated with, (a) 0-order persistent diagram, (b) 1-order persistent diagram, and (c) 2-order persistent diagram.

finish with this part, the analysis of Fig. 9-(a) shows the number of connected components. As we can see, the sig.(e) shows a lot number of connected components, this is related to the strong dispersion of its points. For all 


\begin{tabular}{|l||ccc|}
\hline Model & $\beta_{0}$ & $\beta_{1}$ & $\beta_{2}$ \\
\hline PARMA(2,1) & $\geq 1$ & 2 & 0 \\
PAR(2), $T=20$ & $\geq 1$ & 1 & 0 \\
PAR(2), $T=54$ & $\geq 4$ & 4 & 0 \\
Amplitude Modulated & 1 & 0 & 0 \\
\hline
\end{tabular}

BETTI NUMBERS ACCORDING TO THE CLASS OF THE PROCESSES.

the others, the number of connected components, which is greater than one certainly, is the same; at the exception once again of the sig. $(d)$ for which there is only one connected component. To summarize these results, we propose to update the Table I to Table II.

\section{Information given by the persistent entropy}

Contrary to some authors who applied persistent homology with a single realization of the signal treated [45], our approach is more probabilistic. Indeed, by launching many realizations of the processes for each of the models under study, allows us to describe the probabilistic aspect of persistent homology diagrams. We then investigate the randomness description of the information topology. Subsequently, to our analysis conducted in the previous subsections, we particularly investigate the normalized persistent entropy. We defined the normalized entropy as

$$
H(p)=-\frac{1}{\log \left(N_{p}\right)} \sum_{j=1}^{N_{p}} p_{j} \log \left(p_{j}\right)
$$

where $p_{j}=\frac{l_{j}}{L}$ with $l_{j}=y_{j}-x_{j}, L=\sum_{j=1}^{N_{p}} l_{j}$, and $N_{p}$ the number of points in the persistent diagram. In this way, the maximum entropy is set to 1 whatever the number of points. For any random variables, the entropy reveals the knowledge we may have on its values. For example, the distribution that corresponds to maximum entropy, thus the minimum of information for the realizations of the process, is the uniform distribution. When applied to persistent diagram, it indicates if a particular topological structure emerged from the point cloud. For that case, a maximum entropy will interpret itself as an equal lifetime for all the Vietoris-Rips complexes and will indicate constrained repartitions of the dilation matrices. To stress this point, let us track the evolution of the normalized persistent entropy distribution for the 1-order diagram of a noisy circle when the level of noise increases. The results are reported in Fig. 10. From this figure, the more the central hole is perceptible, the lower the average of the normalized persistent entropy distribution and its shape tends to a uniform distribution. At the opposite, when the noise level is so important that the points seem to be drawn from a uniform distribution on $[0,1]^{2}$, the distribution tends to a Dirac with obviously a high mean value. This is because the points are not free to settle anywhere inside the square. They are constrained by the distribution law which is a purely unit Gaussian process. Let us now see the distribution of the normalized persistent entropy for the processes under study. We have plotted these distributions on Fig. 11. As usual so far, Fig. 11-(a) is associated with the 0order persistent diagram, Fig. 11-(b) with the 1-order diagram and Fig. 11-(c) with the 2-order diagram. Our conclusions for the Fig. 11-(a) are that the persistent entropy for the 0-order diagram allows showing the very weak difference between the processes. All the PAR(2) models tend to a Dirac distribution whereas the PARMA $(2,1)$ model spread off a bit more. The analysis next of the persistent entropy distribution associated with 1-order diagram shows the fact that the sig.(b) and sig.(c) have a low number of points in the diagram and that the loop is clearly identified by only one point with a high lifetime. This seems not to be the same for the sig.(e), for which there are for sure many points in the diagram with more variability. The sig. $(d)$ has nearly the same persistent entropy distribution, which reveals its high volatility and the difficulty to estimate a loop. At least, only the sig. $(d)$ admits a persistent entropy distribution for the 2-order diagram, the values are extremely weak so information about that is almost surely not significant. We have seen with this analysis how the persistent entropy distribution could bring confidence in the determination of the number of connected components or loops. In addition, we have also seen that for the persistent entropy associated with the 0-order diagram, it was possible to discriminate the different processes.

To conclude this part, and based on the results exposed, we are in a position to affirm that the structure of the data point cloud formed by the dilation matrices reveals important intrinsic and hidden properties of the PC random processes. Each of the process class clearly possesses an intrinsic topological structure. We also hope to open doors towards a new way of characterizing spectral properties of non-stationary processes by topological considerations.

\section{CONCLUSION}

The main goal of this work was to give topological features of non-stationary Periodically Correlated (PC) stochastic processes. To do so, we needed first to embed the stochastic process onto a manifold. This has been done by the use of the Dilation theory to connect processes' spectral measure and rotation matrices. Due to the periodicity of the spectral measure of the process 

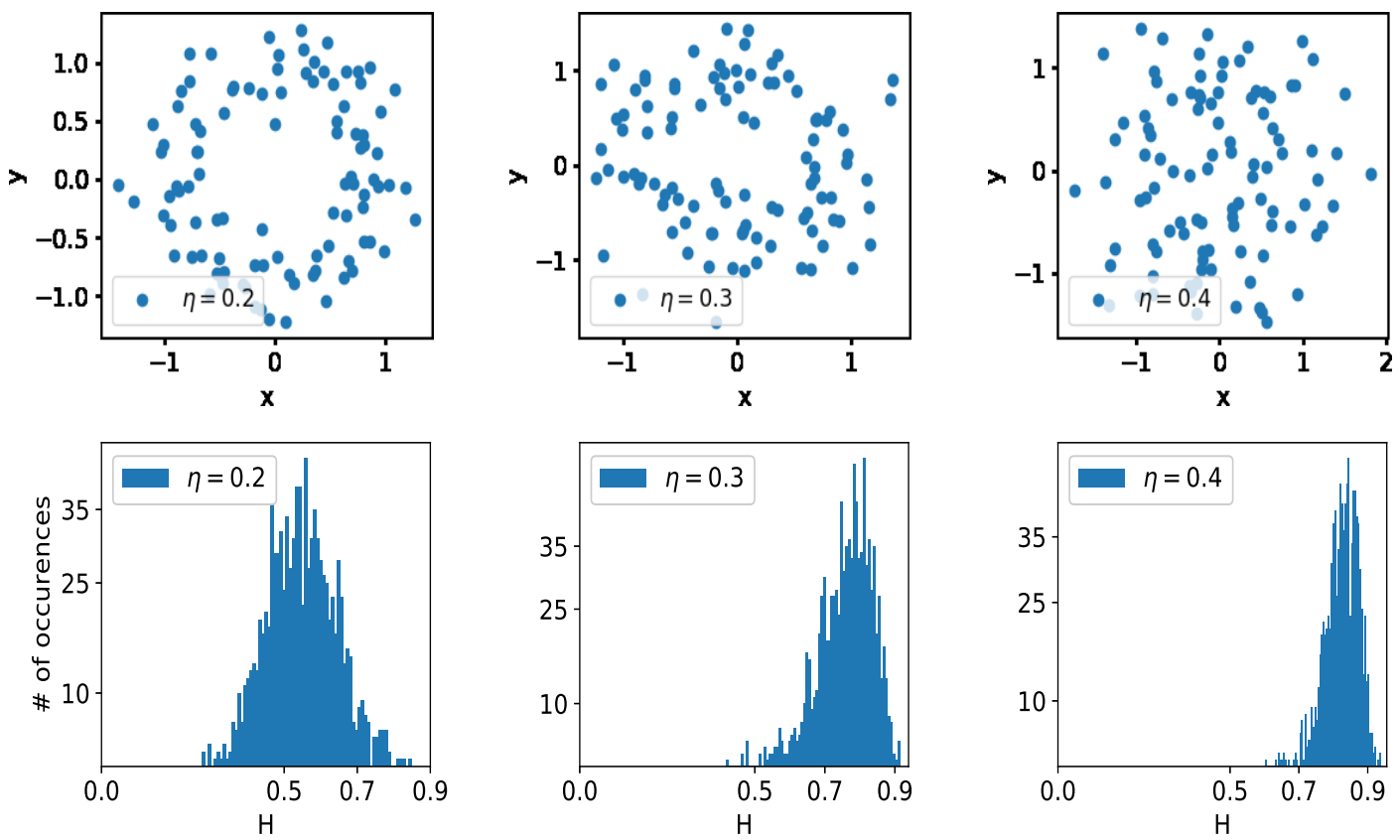

Fig. 10. 500 realizations of a noisy circular data points have been generated for three different noise levels $\eta$. Top: one realization for each of the noise levels, from left to right $\eta=0.2, \eta=0.3, \eta=0.4$. Bottom: the corresponding persistent entropy histograms.

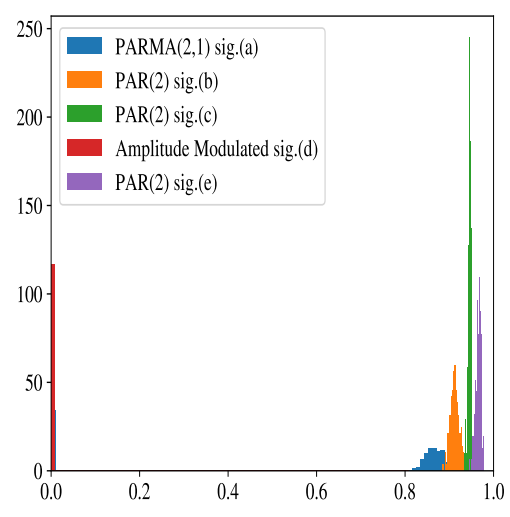

(a)

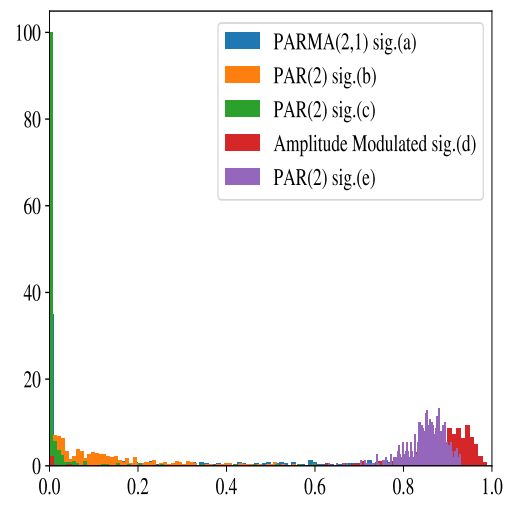

(b)

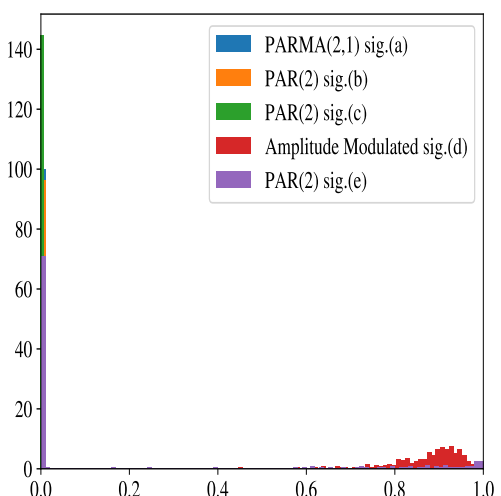

(c)

Fig. 11. Normalized persistent entropy histograms associated with, (a) 0-order persistent diagram, (b) 1-order persistent diagram and (c) 2-order persistent diagram.

studied, the trajectory formed by the dilation matrices on the Lie group is a closed path. Based on this, we have implemented a persistent homology algorithm based on the Vietoris-Rips filtration to obtain useful topological invariants. As expected, the results obtained allowed to clearly differentiate the classes of processes, in such a way that with certain confidence we are able to give the Betti numbers associated with the class of models. For example a PAR(2) model of 1000 samples and a period $T=20$ has Betti numbers $\left(1 \leq \beta_{0} \leq 4\right.$, $\beta_{1}=1, \beta_{2}=0$ ) for its representation on $\operatorname{SO}(3)$. The analysis of the results given by this work also showed that for the representation on $\mathrm{SO}(3)$ the spectral measure of the processes seemed to be oriented. Finally, we would like to mention that the processes studied in this work had finally different persistent diagram which brought out all the necessary information. We may have more difficult scenarios for which other algebraic or deep learning methods could be employed for having a similar interpretation. This is left to future work. 


\section{REFERENCES}

[1] P. Flandrin, ," in Time-Frequency/Time-Scale Analysis, vol. 10 of Wavelet Analysis and Its Applications. Academic Press, 1999.

[2] F. Auger, P. Flandrin, Y.-T. Lin, S. McLaughlin, S. Meignen, T. Oberlin, and H.-T. Wu, "Time-Frequency Reassignment and Synchrosqueezing: An Overview," IEEE Signal Process. Mag., vol. 30, no. 6, pp. 32-41, nov 2013.

[3] A. Napolitano, "Cyclostationarity: New trends and applications," Signal Processing, vol. 120, pp. 385-408, 2016.

[4] H. L. Hurd and A. Miamee, Periodically Correlated Random Sequences: Spectral Theory and Practice, Wiley Series in Probability and Statistics. Wiley, 2007.

[5] L. Yang, M. Arnaudon, and F. Barbaresco, "Riemannian median, geometry of covariance matrices and radar target detection," in Radar Conference (EuRAD), 2010 European, Sept. 2010, pp. 415-418.

[6] F. Barbaresco, "Information Geometry of Covariance Matrix: Cartan-Siegel Homogeneous Bounded Domains, Mostow/Berger Fibration and Fréchet Median," in Matrix Inf. Geom., pp. 199-255. Springer Berlin Heidelberg, Berlin, Heidelberg, 2013.

[7] A. Le Brigant, "Computing distances and geodesics between manifold-valued curves in the SRV framework," Journal of Geometric Mechanics, vol. 9, no. 2, pp. 131-156, 2017.

[8] M. Dugast, G. Bouleux, and E. Marcon, "Representation and characterization of non-stationary processes by dilation operators and induced shape space manifolds," Entropy: From Physics to Information Sciences and Geometry, 2018.

[9] P. Baudot and D. Bennequin, "The homological nature of entropy," Entropy, vol. 17, no. 5, pp. 3253-3318, 2015.

[10] J.P. Vignaux, The structure of information: From probability to homology, Ph.D. thesis, Université Paris Diderot, Sorbonne Paris Cité France, 92017.

[11] T. Constantinescu, Schur Parameters, Factorization and Dilation Problems, Birkhäuser Basel, Basel, 1995.

[12] Elena Celledoni, Markus Eslitzbichler, and Alexander Schmeding, "Shape analysis on Lie groups with applications in computer animation," Journal of Geometric Mechanics, vol. 8, pp. 273-304, 062015.

[13] Alexander Port, Iulia Gheorghita, Daniel Guth, John M Clark, Crystal Liang, Shival Dasu, and Matilde Marcolli, "Persistent topology of syntax," Mathematics in Computer Science, vol. 12, no. 1, pp. 33-50, 2018.

[14] C. Giusti, E. Pastalkova, C. Curto, and V. Itskov, "Clique topology reveals intrinsic geometric structure in neural correlations," Proceedings of the National Academy of Sciences, vol. 112, no. 44, pp. 13455-13460, Nov. 2015.

[15] M. Guillemard and A. Iske, "Signal filtering and persistent homology: an illustrative example," Proc. Sampling Theory and Applications (SampTA'11), 2011.

[16] A. Weron, "Stochastic processes of second order with values in banach space," Transactions of the 7th Prague Conference on Information Theory, Statistical Decision Functions, Random Processes, pp. 567-574, 1977.

[17] H. Hurd, G. Kallianpur, and J. Farshidi, "Correlation and spectral theory for periodically correlated random fields indexed on Z2," Journal of Multivariate Analysis, vol. 90, no. 2, pp. 359-383, Aug. 2004.

[18] H. L. Hurd, "Correlation theory of almost periodically correlated processes," Journal of Multivariate Analysis, vol. 37, no. 1, pp. 24-45, Apr. 1991.

[19] A. Makagon and H. Salehi, "Notes on infinite dimensional stationary sequences," in Probability Theory on Vector Spaces IV, pp. 200-238. Springer, 1989.
[20] B. Sz.-Nagy, C. Foias, H. Bercovici, and L. Kérchy, Harmonic Analysis of Operators on Hilbert Space, Springer New York, New York, NY, 2010.

[21] Hiroshi Takai and Hiroaki Yamada, "A note on the dilation theorems," Proceedings of the Japan Academy, vol. 48, no. 4, pp. 216-220, 1972.

[22] A. E. Frazho, "On Stochastic Bilinear Systems," in Modelling and Application of Stochastic Processes, Uday B. Desai, Ed., pp. 215-241. Springer US, 1986, DOI: 10.1007/978-1-46132267-2_9.

[23] C. Foias and A. E. Frazho, "A Geometric Approach to Positive Definite Sequences," in The Commutant Lifting Approach to Interpolation Problems, number 44 in OT 44 Operator Theory: Advances and Applications, pp. 497-546. Birkhäuser Basel, 1990, DOI: 10.1007/978-3-0348-7712-1_15.

[24] Th Kailath and AM Bruckstein, "Naimark dilations, state-space generators and transmission lines," in Advances in Invariant Subspaces and Other Results of Operator Theory, pp. 173-186. Springer, 1986.

[25] T. Kailath, "Time-Variant and Time-Invariant Lattice Filters for Nonstationary Processes.," outils et modele mathematiques pour l'automatique, l'analyse de systemes et le traitement du signal, vol. 2, 1982.

[26] P. Masani, "Dilations as Propagators of Hilbertian Varieties," SIAM J. Math. Anal., vol. 9, no. 3, pp. 414-456, June 1978.

[27] Aleksander Weron, "Second order stochastic processes and the dilation theory in banach spaces," Ann. Inst. H. Poincaré Sect. $B$, vol. 16, pp. 29-38, 1980.

[28] Gr. Arsene and T. Constantinescu, "The structure of the Naimark dilation and Gaussian stationary processes," Integral Equations and Operator Theory, vol. 8, no. 2, pp. 181-204, Mar. 1985.

[29] D. Timotin, "Prediction theory and choice sequences: an alternate approach," in Advances in invariant subspaces and other results of operator theory, pp. 341-352. Springer, 1986.

[30] M. C. Tseng and V. Ramakrishna, "Dilation Theoretic Parametrizations of Positive Matrices with Applications to Quantum Information,” arXiv:quant-ph/0610021, Oct. 2006, arXiv: quant-ph/0610021.

[31] A. H. Sayed, T. Constantinescu, and T. Kailath, "Recursive Construction of Multichannel Transmission Lines with a Maximum Entropy Property," in Codes, Graphs, and Systems, pp. 259-290. Springer, 2002.

[32] B. Simon, Orthogonal Polynomials on the Unit Circle Part1 and Part 2, vol. 54, American Mathematical Society, 2009.

[33] B. Balaji, F. Barbaresco, and A. Decurninge, "Information geometry and estimation of Toeplitz covariance matrices," in 2014 Int. Radar Conf. oct 2014, pp. 1-4, IEEE.

[34] Gregory Ammar, William Gragg, and Lothar Reichel, "Constructing a unitary Hessenberg matrix from spectral data," in Numerical linear algebra, digital signal processing and parallel algorithms, pp. 385-395. Springer, 1991.

[35] Yuhei Umeda, "Time Series Classification via Topological Data Analysis," Transactions of the Japanese Society for Artificial Intelligence, vol. 32, no. 3, 2017.

[36] Gunnar Carlsson, Tigran Ishkhanov, Vin de Silva, and Afra Zomorodian, "On the Local Behavior of Spaces of Natural Images," International Journal of Computer Vision, vol. 76, no. 1, pp. 1-12, Jan. 2008.

[37] Chad M. Topaz, Lori Ziegelmeier, and Tom Halverson, "Topological Data Analysis of Biological Aggregation Models," PLOS ONE, vol. 10, no. 5, pp. e0126383, May 2015.

[38] Pablo G. Cámara, Arnold J. Levine, and Raúl Rabadán, "Inference of Ancestral Recombination Graphs through Topological Data Analysis," PLOS Computational Biology, vol. 12, no. 8, pp. e1005071, Aug. 2016. 
[39] Alexander Port, Iulia Gheorghita, Daniel Guth, John M. Clark, Crystal Liang, Shival Dasu, and Matilde Marcolli, "Persistent Topology of Syntax," Mathematics in Computer Science, vol. 12, no. 1, pp. 33-50, Mar. 2018.

[40] N. Otter, M. Porter, U. Tillmann, P. Grindrod, and H. Harrington, "A roadmap for the computation of persistent homology," EPJ Data Science, vol. 6, no. 17, pp. 1-38, 2017.

[41] Edelsbrunner, Letscher, and Zomorodian, "Topological Persistence and Simplification," Discrete \& Computational Geometry, vol. 28, no. 4, pp. 511-533, Nov. 2002.

[42] Herbert Edelsbrunner and Dmitriy Morozov, Persistent Homology: Theory and Practice, 2013.

[43] A. Zomorodian and G. Carlsson, "Computing Persistent Homology," Discrete Comput Geom, vol. 33, no. 2, pp. 249-274, Feb. 2005

[44] N. Boumal, "Interpolation and regression of rotation matrices," in Geometric Science of Information, pp. 345-352. Springer, 2013.

[45] Jose A Perea and John Harer, "Sliding windows and persistence: An application of topological methods to signal analysis," Foundations of Computational Mathematics, vol. 15, no. 3, pp. 799-838, 2015.

[46] M. Piangerelli, M. Rucco, L. Tesei, and E. Merelli "Topological classifier for detecting the emergence of epileptic seizures" BMC Research Notes, vol. 11

[47] E. Merelli, M. Piangerelli, M. Rucco, and D. Toller A topological approach for multivariate time series characterization: the epileptic brain "In Proceedings of the 9th EAI International Conference on Bio-inspired Information and Communications Technologies (formerly BIONETICS)” pp. 201-204, May, 2016. 